Three-dimensional electrical impedance tomography applied to a metal-walled filtration test platform

This article has been downloaded from IOPscience. Please scroll down to see the full text article.

2004 Meas. Sci. Technol. 152263

(http://iopscience.iop.org/0957-0233/15/11/012)

View the table of contents for this issue, or go to the journal homepage for more

Download details:

IP Address: 130.88.118.249

The article was downloaded on 14/06/2011 at $14: 47$

Please note that terms and conditions apply. 


\title{
Three-dimensional electrical impedance tomography applied to a metal-walled filtration test platform
}

\author{
J L Davidson ${ }^{1}$, L S Ruffino ${ }^{2}$, D R Stephenson ${ }^{2}$, R Mann², \\ B D Grieve ${ }^{3}$ and T A York ${ }^{1}$ \\ ${ }^{1}$ Industrial Process Tomography Group, Department of Electrical Engineering and \\ Electronics, UMIST, Manchester, M60 1QD, UK \\ ${ }^{2}$ Industrial Process Tomography Group, Department of Chemical Engineering, UMIST, \\ Manchester, M60 1QD, UK \\ ${ }^{3}$ Syngenta Ltd, Technology and Projects Department, Huddersfield, HD2 1FF, UK
}

Received 26 May 2004, in final form 26 August 2004

Published 8 October 2004

Online at stacks.iop.org/MST/15/2263

doi:10.1088/0957-0233/15/11/012

\begin{abstract}
The first true three-dimensional image reconstructions from a metal-walled vessel using electrical impedance tomography (EIT) are presented. Two image reconstruction techniques have been applied via relatively sophisticated FEM modelling of a bespoke laboratory test vessel from which data have been obtained using an EIT instrument designed to intrinsically safe requirements. A generalized Tikhonov regularization method is compared with the linear back-projection (LBP) technique. Subsequent image reconstructions strongly suggest that the LBP method when applied to a metal-walled vessel is highly sensitive to the level of detail within the FEM model. By comparison, the regularized technique is far less sensitive to the complexity of the modelled geometry. Additionally, unlike the LBP method, the regularization technique has been successful in accurately reconstructing multiple inhomogeneities within an aqueous system. A further experiment has shown similar sensitivity in a wetted powder-based system. It is concluded that EIT via a regularized difference imaging approach has significant potential for detecting 3D malformations and non-uniformities in industrial pressure filtration systems.
\end{abstract}

Keywords: EIT, image reconstruction, filtration

(Some figures in this article are in colour only in the electronic version)

\section{Introduction}

The present status of electrical tomography is such that the many successful laboratory-based studies [1-4] and pilot plant demonstrators are generating interest in developing the technology to production plant level across the chemical processing industry. The ProceMon (Process Tomography for Monitoring Industrial Pressure Filters) project has taken the first major steps in this direction by using the first certified Intrinsically Safe (I.S.) EIT system [5] and has shown that a conventional circumferential sensor arrangement is not a pre-requisite for tomography [6]. This paper investigates three-dimensional image reconstruction using real measurements obtained from a metal-walled laboratory test platform, which can be considered to be comparable to a large-scale industrial filtration unit.

The aim of a tomography system is to determine the material distribution within a volume of interest by acquiring measurement data via sensors positioned on the periphery of the volume. However, for image reconstruction, this usually implies far fewer independent measurements compared with the number of pixels in the image space, and therefore, the 
problem is underdetermined. Additionally, in the case of soft fields such as those present in EIT, the electric field is perturbed as a consequence of encountering regions of different conductivities or permittivities. For these reasons, image reconstruction is a non-trivial problem and no single explicit solution method exists. Consequently, a number of reconstruction algorithms have been developed for soft-field problems and can be found, for instance, in the Proceedings of the World Congress on Industrial Tomography [7-9], Process Tomography: Principles, Techniques and Applications [10] or in special issues of Measurement Science and Technology [11-13].

In the past, efforts by the tomography community have tended to focus on 2D image reconstruction [14-16] or three dimensions interpolated from $2 \mathrm{D}$ planes $[17,18]$ as opposed to true $3 \mathrm{D}$ imaging. This has been primarily due to the general trend towards fast data collection hardware by using a small number of sensors either in a single cylindrical plane or combination of planes and neglecting the out-ofplane field contributions in the interests of producing rapid image reconstruction. Additionally, until recently, the greater computational cost requirements have prohibited the solution to $3 \mathrm{D}$ tomography problems.

Early 3D algorithms were primarily developed with medical EIT in mind and include those of Gobel et al [19,20] and Metherall et al [21]. However, in the field of industrial process tomography, with the most notable exception of Pinheiro [22], dedicated 3D algorithms are somewhat scarce. More recently, the application of image reconstruction to a specific tomography problem has become simplified by the introduction of user-defined functions implemented from Electrical Impedance and Diffuse Optical Reconstruction Software (EIDORS). This is a tomography tool-suite written in MatLab script and was first introduced in 2D form by Vauhkonen et al [23] in 2000. More recently, EIDORS has been further enhanced into a 3D version by Polydorides and Lionheart [24] for application to insulating wall vessels. Additionally, the code has been adapted for vessels with conducting walls by Grieve [25] and York et al [6] but this has only been previously tested using simulated data as opposed to real measurements. For this reason, this work investigates the performance of $3 \mathrm{D}$ image reconstruction algorithms for real data obtained under known conditions from a bespoke laboratory test filter and using an EIT instrument built to I.S. specifications. Later sections give a description of the methods used for reconstructing images for a water-based system and an experiment using a filter cake formed with a standard filter aid material.

\section{Motivation behind the research}

Traditionally, monitoring in the process industry has been limited to either visual inspection or single point product sampling where product uniformity is assumed. EIT has the advantage of providing imaging of optically opaque materials and gives the opportunity of providing quantitative, real-time data on chemical media within a full-scale industrial process, such as filtration, without the need of process interruption. The major potential benefits are two-fold; firstly, it is possible to gain an insight into the actual process and secondly, since EIT lends itself to on-line monitoring, the opportunity to develop closed loop control systems. The overall anticipated effects are improvements in product yield and uniformity, minimized input process material, reduced energy consumption and environmental impact and the lowering of occupational exposure to plant personnel.

The ProceMon project [26, 27] uses two prototype systems commissioned at the Syngenta Huddersfield Manufacturing Site, UK, on established full-scale production units. The first is located on a $36 \mathrm{~m}^{3}$ pressure filter unit, shown in figure 1 , and uses 24 sensing electrodes arranged in a single plane within the filter and controlled by I.S. EIT hardware. The work reported in this paper demonstrates image reconstruction techniques applied to a laboratory test vessel using a similar but smaller-scale planar electrode system. The experimental arrangement serves as a highly useful test platform for comparing image reconstruction techniques in a manner that is analogous to the physical and chemical processes present within the large-scale industrial pressure filtration demonstrator on the ProceMon project.

\section{EIT and the conducting boundary measurement strategy}

The first applications of EIT only considered electrode arrangements operating within vessels having insulating walls and using the adjacent measurement strategy. In this case, small ac currents are typically injected across a neighbouring pair of electrodes, whilst voltage measurements on successive adjacent electrode pairs are acquired sequentially by multiplexing. This process is then repeated for all possible unique combinations to give a complete set or frame of measurements. However, the majority of industrial process vessels have conducting walls and therefore, provide an additional current sink during the measurement process. This gives rise to both a reduced sensitivity in the bulk of the material and increased difficulty in obtaining stable measurements referenced to the injected currents. To overcome this problem, the conducting boundary strategy was developed [28-30], which considers each electrode to sequentially act as a current source whilst the whole of the metallic vessel behaves as a grounded current sink. In this strategy, all the voltage measurements are referenced to the same earth potential of the conducting boundary. The basic principle is shown in figure 2 for a 16 electrode system.

The number of unique measurements, $N$, in the conducting boundary or 'metal wall' strategy can be defined as follows:

$$
N=\frac{n(n-1)}{2}
$$

where $n$ is the total number of electrodes. In the present study, a total of eight electrodes are used giving rise to 28 unique measurements.

\section{Image reconstruction}

The process of image reconstruction for EIT requires solving a set of simultaneous equations relating every part of the image space to all the measurement data. To achieve this, the first step involves injecting a known current into a defined 
(a)

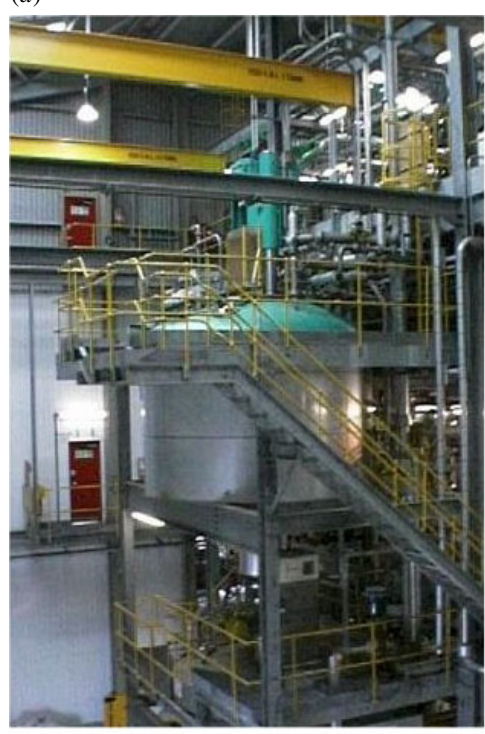

(b)

(c)
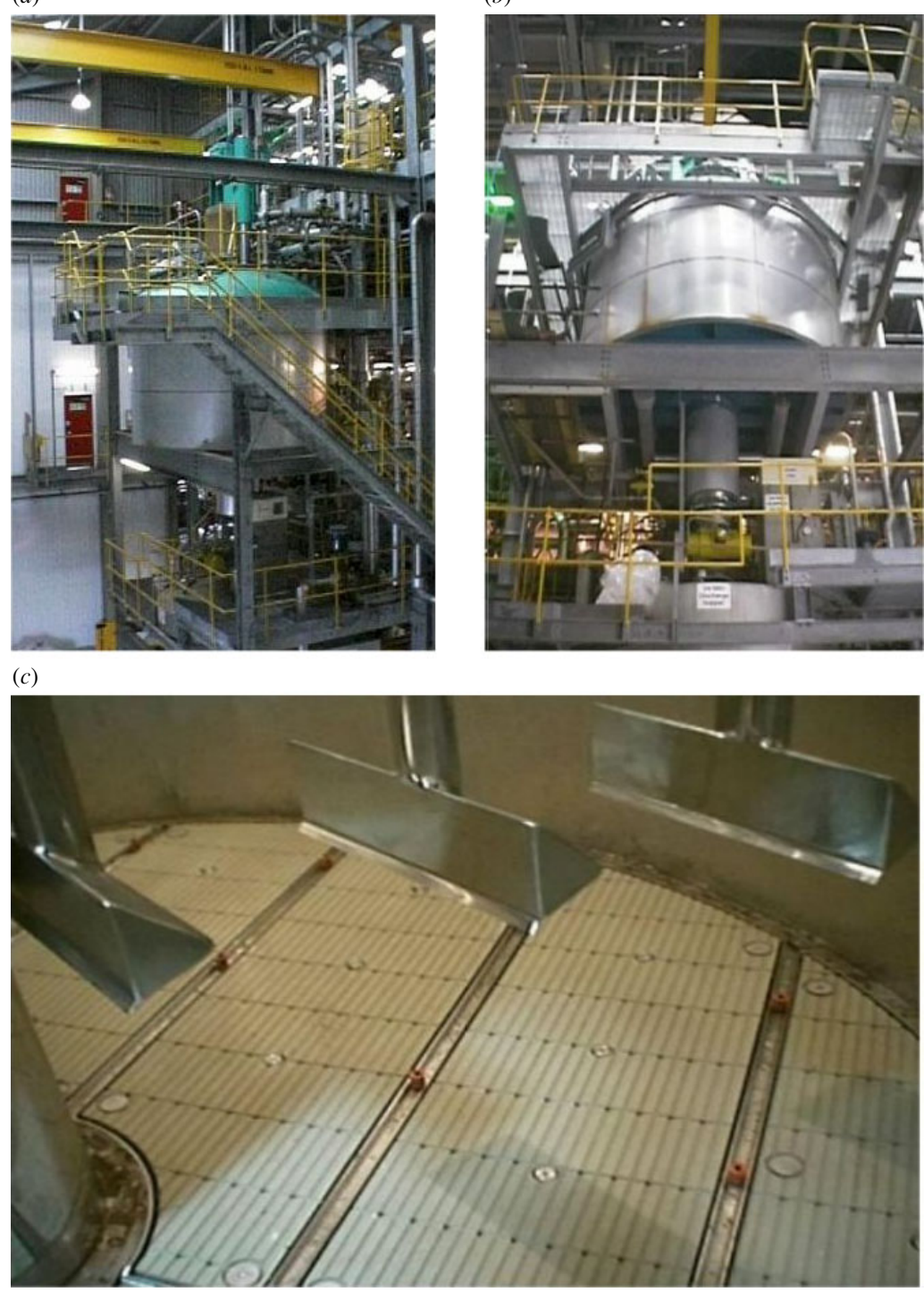

Figure 1. Views of the $36 \mathrm{~m}^{3}$ ProceMon pressure filter; $(a)$ and $(b)$ external views and $(c)$ internal view showing agitator blades and fixing bases for supporting the filter cloth.

homogeneous conductivity, $\sigma$, and calculating the scalar potential, $\phi$, throughout the geometry of interest. In this case, the finite element method (FEM) can be used to solve the Poisson equation:

$$
\nabla \cdot \sigma \nabla \phi=0
$$

and is done so for all the unique electrode measurement pairs. From this, it is possible to extract a column vector of the theoretical voltages on all the electrodes for each current injection by using the complete electrode model of Cheng et al [31]. This considers the voltage on the $i$ th electrode, $V_{i}$, to be determined by the sum of the potentials on the boundary surface underneath that electrode and the potential drop across the electrode's contact impedance, $Z_{c}$, which exists between the electrode and the filling medium. In this study, only low frequency measurements have been acquired, and therefore, the complete electrode model can be described as:

$$
V_{i}=\phi+Z_{c} \sigma \frac{\partial \phi}{\partial v}
$$

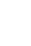




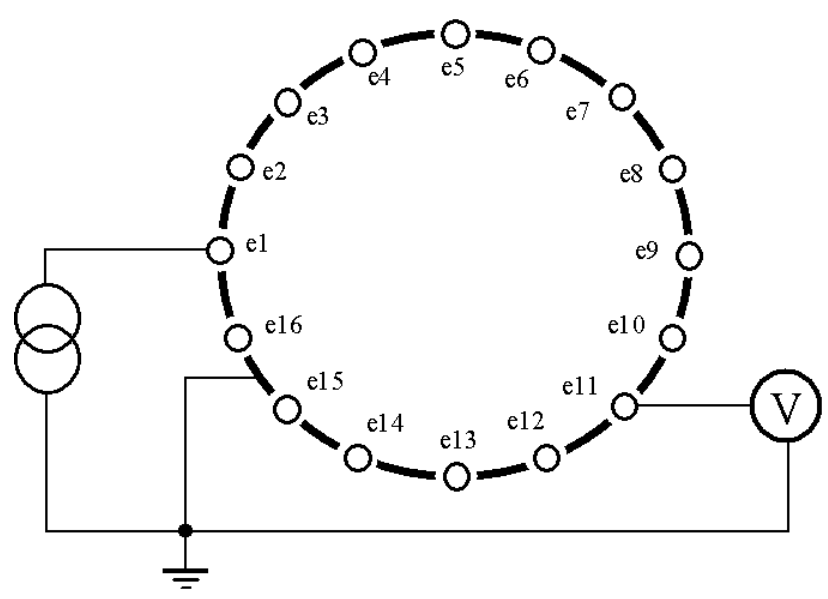

Figure 2. Principle of the conducting boundary strategy for a 16 electrode system.

widespread in EIT, the problem is linearized. This study assumes a linear approximation and attempts to determine the unique conductivity distribution for a set of measured voltages as described by the following equation:

$$
\sigma=J^{-1} V \text {. }
$$

However, a direct solution to equation (6) does not exist since $J$ is an ill-conditioned matrix, and therefore, an alternative approach needs to be adopted. A number of methods for calculating the inverse solution exist and have been rigorously reviewed by Lionheart [33, 34]. This work has investigated two non-iterative methods. The first technique is the linear back-projection (LBP) method, which approximates equation (6) to the following:

$$
\sigma \approx S^{T} V_{m}
$$

where $S^{T}$ is the transpose of the normalized Jacobian and $V_{m}$ represents the measurement voltage vector normalized to the actual injected electrode currents. The LBP is computationally straightforward to implement and is a popular method for image reconstruction but this study is the first time the technique has been applied to a metal-walled vessel comprising a planar electrode arrangement and using actual measurements as opposed to simulated data.

For comparison with the LBP method, a second image reconstruction technique has been employed and is based on a generalized Tikhonov regularization method. The regularization has the advantage of improving the stability and reliability of the inverse solution [35]. The regularized method can be described as follows:

$$
\sigma=\left(S^{T} \cdot S+\alpha I\right)^{-1}\left[S^{T}\left(V_{m}-V_{f r}\right)\right]
$$

where $I$ is a unity matrix, $\alpha$ is a scalar smoothing or regularization parameter and $V_{f r}$ is the predicted voltage vector on the electrodes from the forward solution. Traditionally, equation (8) is used in an iterative manner (e.g. Yorkey et al [36]) which converges on a final solution based on an initially estimated conductivity, by updating the conductivity in response to the difference in the measurement and predicted voltage vectors. However, the method requires the computation of the product of $S^{T}$ and $S$, which for large matrices will be limited by the availability of memory addressing. Additionally, the performance of an iterative regularized method depends on both the initial selection of $\alpha$ and the step size of $\alpha$ per iterative step.

This study adopts a difference imaging technique applied to both the LBP and regularized methods, where each method is used in a single shot non-iterative manner. In this case, the sensitivity matrix is pre-calculated for the geometry of the test vessel from the forward solution data and is used to give an absolute reference image from real measurements obtained for a homogeneous system. This is repeated for real measurements obtained from experiments involving the addition of an inhomogeneity or inhomogeneities as to give the absolute image using the same pre-calculated sensitivity matrix. If the vector describing the difference in the reconstructed conductivity between the two cases is $\sigma_{\text {diff, }}$ then:

$$
\sigma_{\text {diff }}=\sigma_{\text {phantom }}-\sigma_{\text {ref }}
$$

where $\sigma_{\text {phantom }}$ is the calculated absolute conductivity for the inhomogeneity or 'phantom' and $\sigma_{\text {ref }}$ is the calculated absolute conductivity for the homogeneous or 'reference' case.

The non-iterative difference imaging approach has distinct advantages when applied to EIT. In particular, the conductivity depends on both the bulk conductivity and the contact impedance term shown in equation (3), which occurs at the interface between the electrode surfaces and the bulk material. This is an unknown parameter but may be expected not to change significantly from the reference case on the introduction of an inhomogeneity, providing the inhomogeneity is not in contact with the electrode surface and that the bulk properties of the reference material remain unchanged. For this reason, if the same contact impedance is assumed for both the reference and phantom cases, then the difference imaging approach may eliminate the uncertainty in selecting this parameter. Similarly, in the case of the regularized method, by using a non-iterative difference approach, the importance of selecting a suitable value of $\alpha$ for convergence is eliminated and the product of $\alpha$ and $I$ can therefore be considered analogous to a simple scaling factor in ordinary algebra.

\section{Laboratory test filter platform}

The test filter platform uses a multiplexing switching unit, which is of the same design as the I.S. systems linked to commercial pressure filters on ProceMon. In this case, the hardware can switch a maximum of eight electrodes in driving, receiving or floating mode via I.S. barriers. Measurements were obtained using a Hewlett-Packard 4284A precision LCR meter and both the switching hardware and LCR meter are controlled using a standard PC running bespoke data capture software. This type of arrangement has been described in more detail elsewhere [5].

The test filter itself is a stainless steel vessel measuring $0.5 \mathrm{~m}$ in diameter and has a capacity of approximately 80 litres. The filter is fitted with a polypropylene base insert, which provides support for both the filter cloth and electrode array. This base has two concentric rings of drainage holes for the filtration process and the cloth is fixed via a stainless steel clamping ring or 'hold-down' bar. Sensing electrodes are based on a design used in the $36 \mathrm{~m}^{3}$ filter on ProceMon and are manufactured from hastelloy and PTFE. Additionally, in the interests of comparability, the wiring between the multiplexer 
(a)

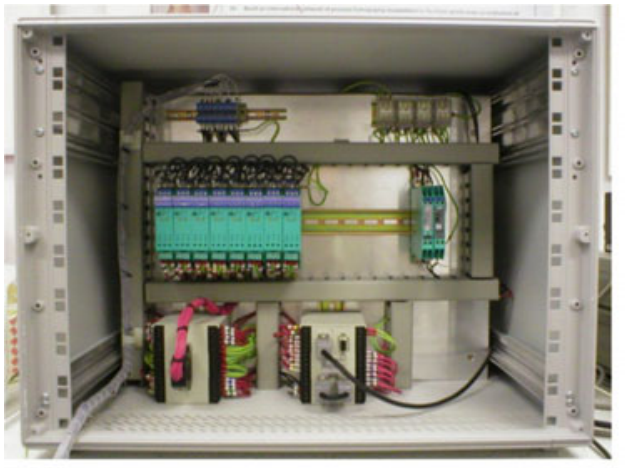

(c)

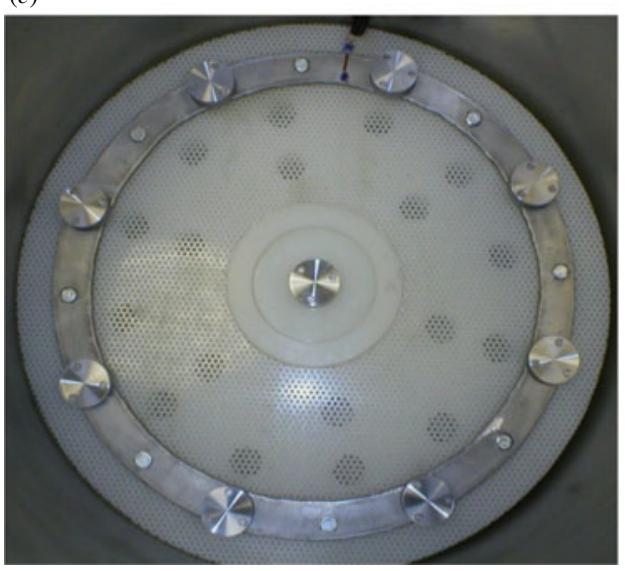

(b)

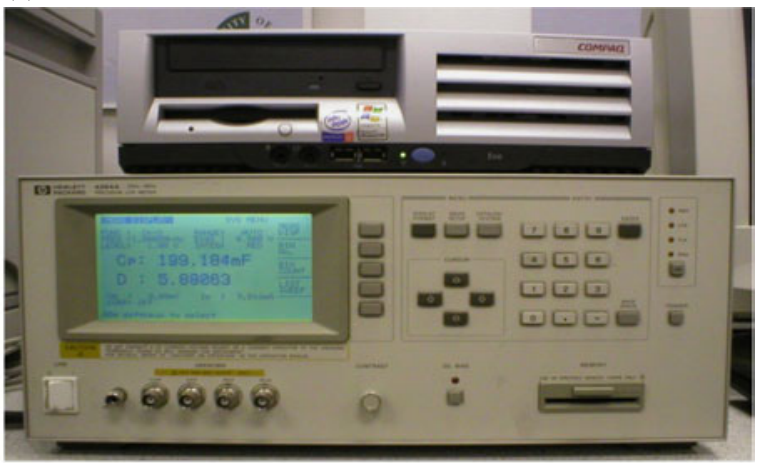

(d)

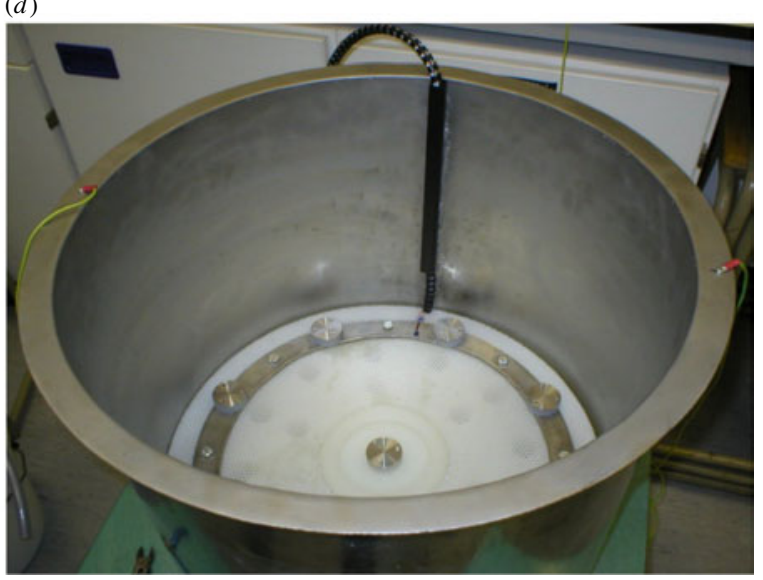

Figure 3. Laboratory test filter platform; (a) BigSwitch multiplexer, $(b)$ impedance analyser, $(c)$ interior of test filter showing hold-down bar with one central and eight outer electrodes, $(d)$ general view of test filter.

and the electrodes is the same type as used on ProceMon. The vessel can be fitted with eight electrodes positioned on the hold-down bar and a further central electrode. Views of the test filter platform are shown in figure 3.

\section{Modelling of the test filter}

The open source software, NetGen [37], has been used to create meshes of the test filter in order to describe the equation nodes for solving both the forward and inverse problems. For accurate calculation of the forward solution it is necessary to accommodate the field perturbations present in the system by including all the conducting surfaces, which will have a strong influence on the electric field distribution. However, over-modelling is unwanted for two reasons. Firstly, for a fixed measurement vector, increasing the number of mesh elements will make the problem further underdetermined and possibly mathematically intractable to solve for a particular reconstruction algorithm. Secondly, large meshes increase the required computational effort. For these reasons, three geometrical models of the filter were generated and are shown in figure 4.

The first is shown in figure 4(a) and models the electrode positions on the filter cloth hold-down bar and comprises a mesh of approaching 25000 tetrahedra. The second model (figure $4(b)$ ) is the same as the first model and is similar in mesh size but also considers the drainage holes in the polypropylene base and the fixing nuts on the hold-down (a)

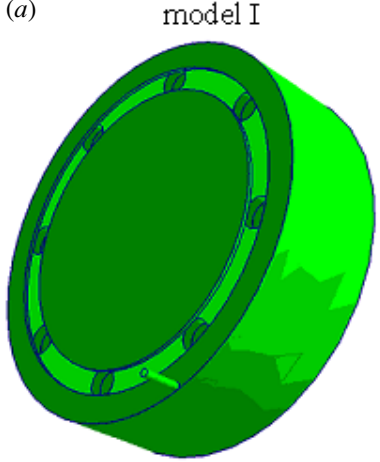

(c)

model III
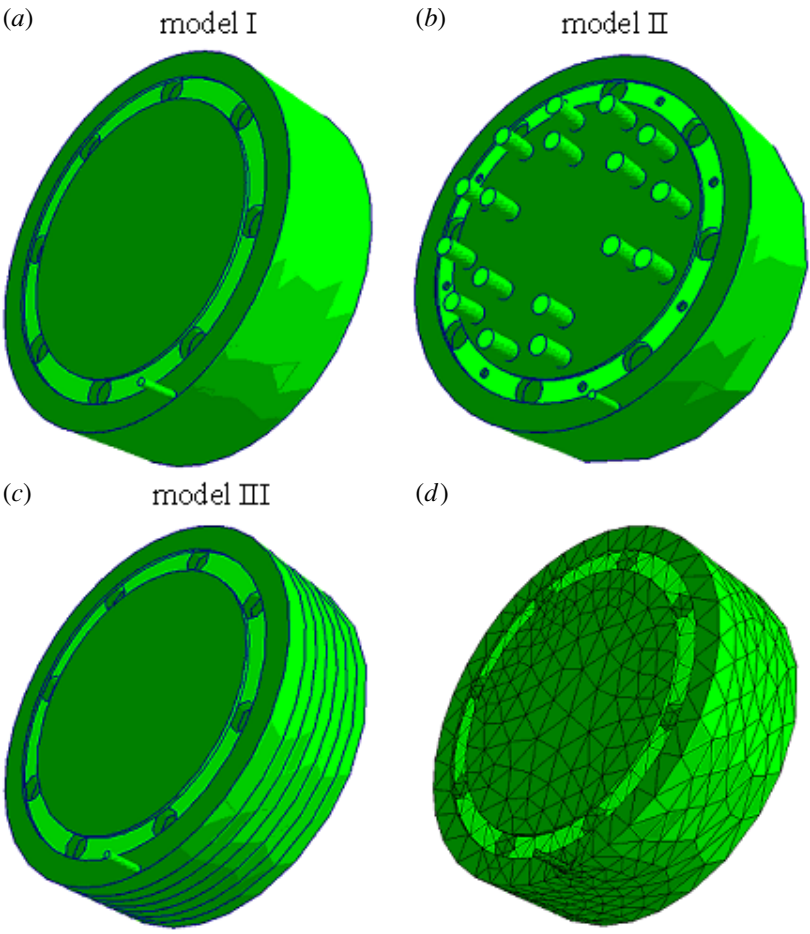

(d)

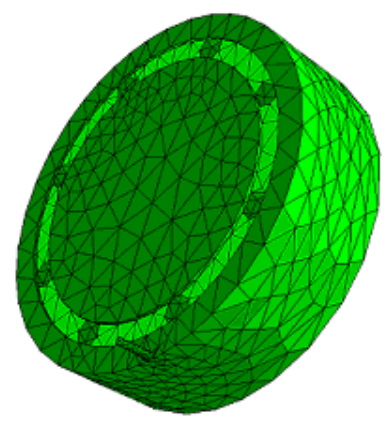

Figure 4. FEM modelling of the laboratory test filter; $(a)$ and $(b)$ are the simple and complex geometrical models used for LBP image reconstruction, $(c)$ stacked layered model used for both LBP and regularized reconstructions and $(d)$ mesh associated with $(c)$. 
bar. In both of these models the outer wall of the filter is modelled as a continuum and, like the hold-down bar, is defined as a grounded boundary condition. As a result of memory addressing, models comprising such large numbers of tetrahedra cannot be used to solve the inverse problem by the described regularized method using only a modest PC. For this reason, a third model, shown in figure 4(c), comprising fewer elements, only considers the electrode positions on the holddown bar but unlike the first model uses a layered approach to construct the complete depth of the filter. In this case, six discs of equal depth are stacked onto a base layer to achieve a more uniform mesh density throughout the bulk volume of the filter. This model comprises a mesh size of the order of 4500 tetrahedra and is used for both LBP and regularized difference imaging. All models described the outer conducting wall of the vessel to a depth of $10 \mathrm{~cm}$ from the polypropylene base insert. Additionally, for all models, a narrow diameter cylinder on the underside of the filter is used to model the small current path to ground via the filling material surrounding the grouping of the electrode connecting wires to the underside of the polypropylene base. The position of this cylinder is approximately adjacent to electrode 2 for the electrodes traversed clockwise when viewed from above, and although, this current leakage path is likely to be negligible, the point is useful for defining the orientation of the filter in subsequent 3D viewing. Also in the interests of clarity, the three geometrical models are subsequently referred to as models I, II and III as indicated in figure 4.

\section{Experimental details}

Several simple experiments were performed in order to test the effectiveness of the described image reconstruction methods. These experiments have considered, firstly, a water-based system and, secondly, a finely divided wetted powdered system. All the measurement data were obtained at approximately $10 \mathrm{kHz}$ using the described multiplexing system with the analogue signals furnished via I.S. barriers between the electrodes and LCR impedance analyser. Additionally, the test vessel was configured to the eight electrodes on the holddown bar and the injected current was impedance limited by the LCR meter to a maximum of the order of $150 \mu \mathrm{A}$.

For the aqueous system, inhomogeneities comprised lowdensity expanded foam, typical of the artificial sponges used for both domestic cleaning and personal hygiene. In initial experiments, the measured conductivity of the reference water was approximately $8.5 \mathrm{mS} \mathrm{m}^{-1}$. The first experiment involved the addition of a single sponge at one of either two separate positions shown schematically in figure 5(a). In this case, prior to experiment, each sponge was soaked for several minutes in a second vessel containing water from the same source and having a similar conductivity as the reference water in the test filter itself. However, since the sponges are polymer based, in these experiments, the phantoms can be considered to be less conducting with respect to the reference. The experiment was repeated for two sponges used as phantoms simultaneously, but this time one sponge was immersed in common salt solution having a conductivity of approximately $150 \mathrm{mS} \mathrm{m}^{-1}$ prior to being added to the test filter. In this second type of experiment, the sponge soaked in the salt solution can
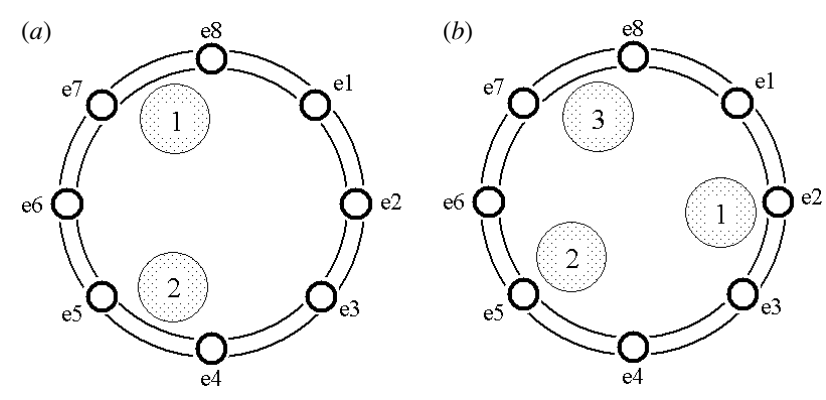

Figure 5. Schematic representation of the phantom positions used in the aqueous based system: $(a)$ for the single and double phantom experiments and $(b)$ for the triple phantom experiment.

be considered to be conducting compared with the background reference. An additional experiment used three sponges acting as phantoms simultaneously. In this case, the reference water conductivity was approximately $50 \mathrm{mS} \mathrm{m}^{-1}$ and the sponges were positioned as shown in figure $5(b)$. Prior to phantom measurements, as in earlier experiments, the sponges were soaked in salt solution, two in a conductivity of $1000 \mathrm{mS} \mathrm{m}^{-1}$ and the third at a conductivity of $10 \mathrm{mS} \mathrm{m}^{-1}$.

For the wetted powder-based system, a standard inert filter aid material (Clarcel-Flo supplied by Atofina UK, Ltd) was used to construct a filter cake. The nominal particle size was of the order of $200 \mu \mathrm{m}$ and this type of material is widely used by industrial filtration unit and filter cloth manufacturers for assessing the performance of both filter vessels and filter fabric. The cake was constructed over several days from an initial water and filter aid 'slurry'. The formation process involved cycles of slurry mixing followed by drying periods in such a way as to produce a relatively smooth and homogeneous cake. After forming the cake, a cylindrical phantom measuring approximately $9 \mathrm{~cm}$ in diameter was produced by the removal of a small volume of the cake material.

For image reconstruction, the calculations were performed using a modest PC with a P4 CPU operating at $2.66 \mathrm{MHz}$ and $512 \mathrm{MB}$ of RAM. Typical total processing time for the forward solution, sensitivity analysis and inverse solution varied between a few seconds for the LBP method to several minutes for the non-iterative regularized technique.

\section{Image reconstruction in the aqueous system}

The sensitivity of the LBP method to FEM modelling can be clearly seen in figure 6 , which shows $x-y$ planes extracted at different positions from the $3 \mathrm{D}$ image reconstruction and referenced to the electrode plane itself. All the axis units are in metres with the $x$ and $y$ describing the radial position from the centre of the filter and the $z$-axis giving the height above the electrode plane. In this figure, reconstruction used geometrical models I and II of the test filter as shown in figures $4(a)$ and $(b)$ respectively. The lowest planes of figures $6(c)$ and $(d)$ arise as a consequence of the modelled drainage holes of the filter vessel in geometrical model II.

Despite a large mesh size of 25000 elements, the difference LBP method fails to reconstruct the single inhomogeneity in either position 1 or 2 for geometrical model I. This is in contrast with the reconstructions using the more complex modelling of model II (figures $6(c)$ and $(d)$ ), which 

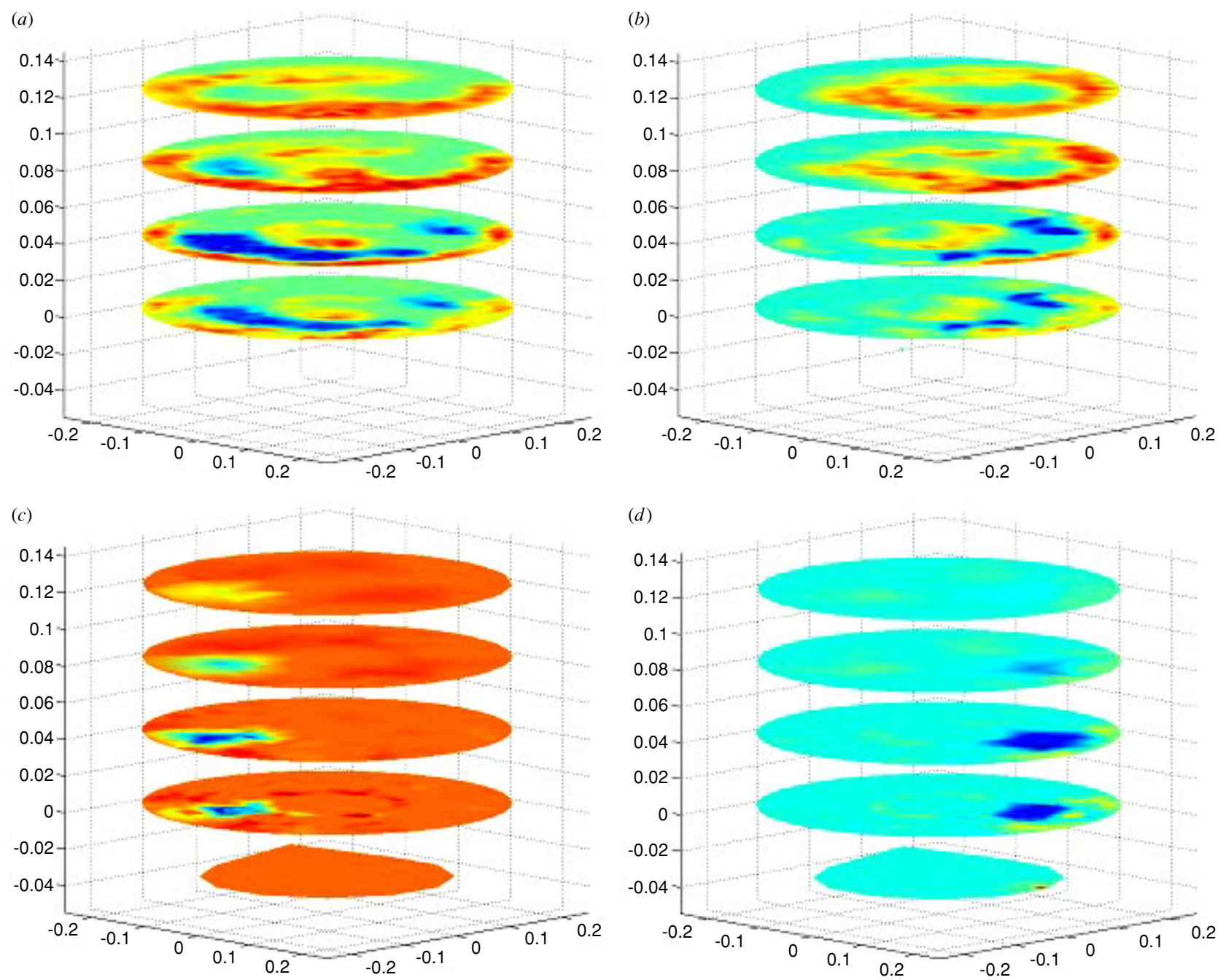

Figure 6. Difference LBP images for the aqueous system using two of the described FEM models each of approximately 25000 elements; $(a)$ and $(b)$ use geometrical model I for single sponges in positions 1 or 2 respectively, $(c)$ and $(d)$ reconstruction using geometrical model II from the same measurement data. All axis labels are in metres and have been omitted for clarity.

more accurately describes the structural components of the test vessel responsible for either perturbing the field or providing additional current paths to ground. Using model II, the associated reconstructions predict the inhomogeneities at both the correct locations and the approximate diameters of the physical objects. Additionally, for both sponge positions, the relative conductivity is predicted as expected, i.e. the inhomogeneities are of lower conductivity compared with the reference background. These reconstructions are the first 3D images from a metal-walled vessel using a planar electrode arrangement, and so are highly encouraging, however, the experiments suggest the need for an accurate forward solution for success of the LBP technique when applied to the conducting boundary strategy. This imposes considerable limitations on the technique, since in modelling real industrial vessels it would be necessary to define all the internal metallic components such as dip legs, agitator blades, inlet ports etc in order to calculate an accurate forward solution.

The improvement in image reconstruction provided by the regularization compared with LBP can be clearly seen in figure 7 , which gives a comparison of regularized method and the LBP for the simple mesh described by the stacked layer geometry of model III as shown in figure 4(c). The LBP fails to reconstruct the inhomogeneities using this significantly smaller mesh, unlike the regularized method, which for the same measurement data accurately predicts the phantoms despite the use of a lower number of elements compared with the reconstructions of the previous figure. Additionally, the regularized method has been successful in correctly locating the inhomogeneities throughout the entire modelled $z$-axis without any significant loss of sensitivity, which represents a $z$-axis to diameter ratio of approximately 0.2 , i.e. approaching a comparable geometry to that of a typical industrial pressure filter. This is a significant result considering the image reconstruction has only used a single planar ring of eight electrodes producing only 28 unique measurements.

For the test filter, the described LBP method failed to successfully predict the presence of double and triple simultaneous inhomogeneities, irrespective of the FEM mesh used for image reconstruction. However, the regularized technique is sufficiently sensitive, under the tested conditions, to locate two sponge phantoms as can be seen in figure 8, showing the more conducting sponge at position 1 and the less conducting sponge at position 2 as described in figure $5(a)$. 

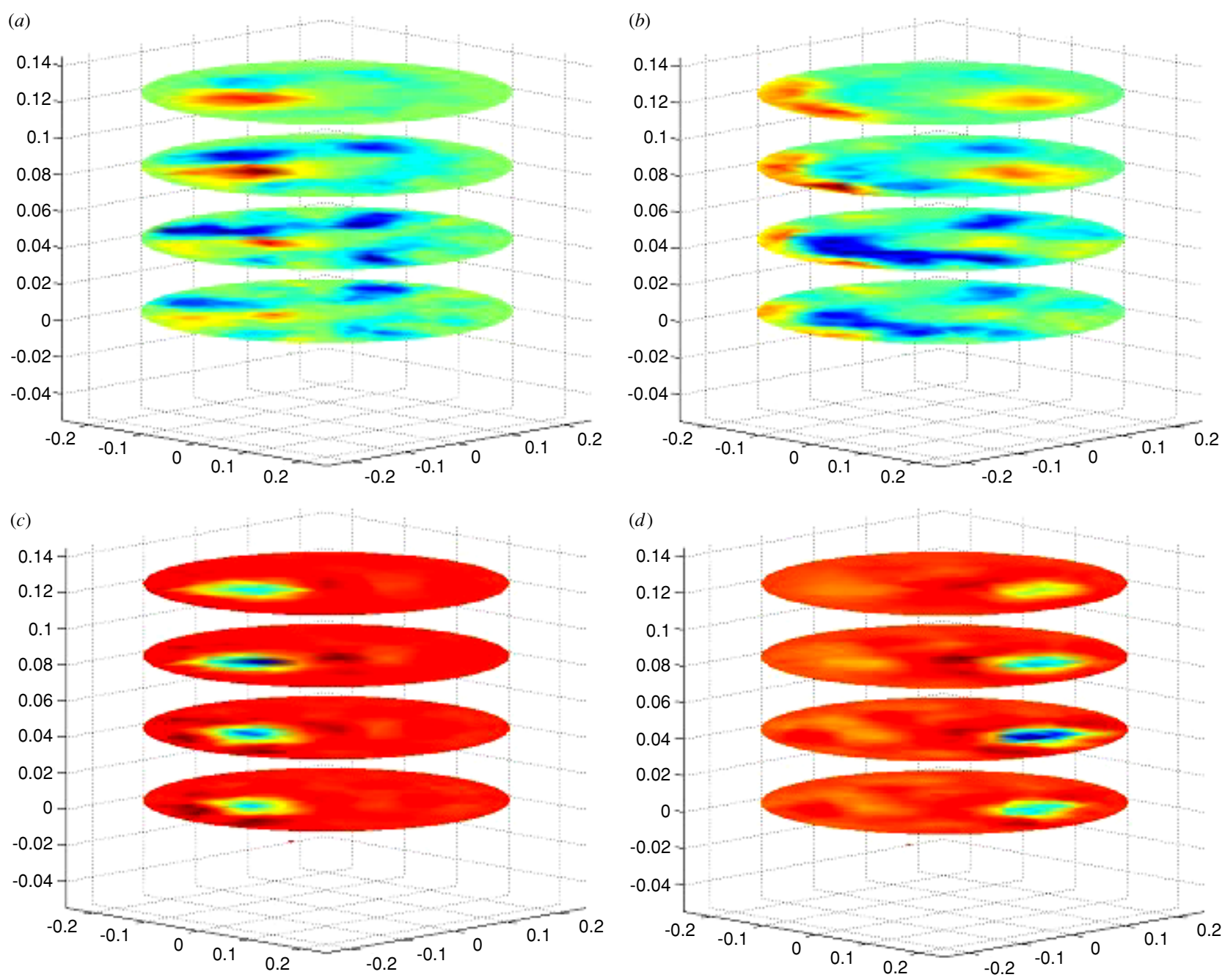

Figure 7. Comparison of LBP and regularized methods using geometrical model III comprising approximately 4500 elements: (a) and $(b)$ use the described LBP method for single sponges in positions 1 or 2 respectively, $(c)$ and $(d)$ reconstruction using the same measurement data and the regularized method. All axis labels are in metres and have been omitted for clarity.
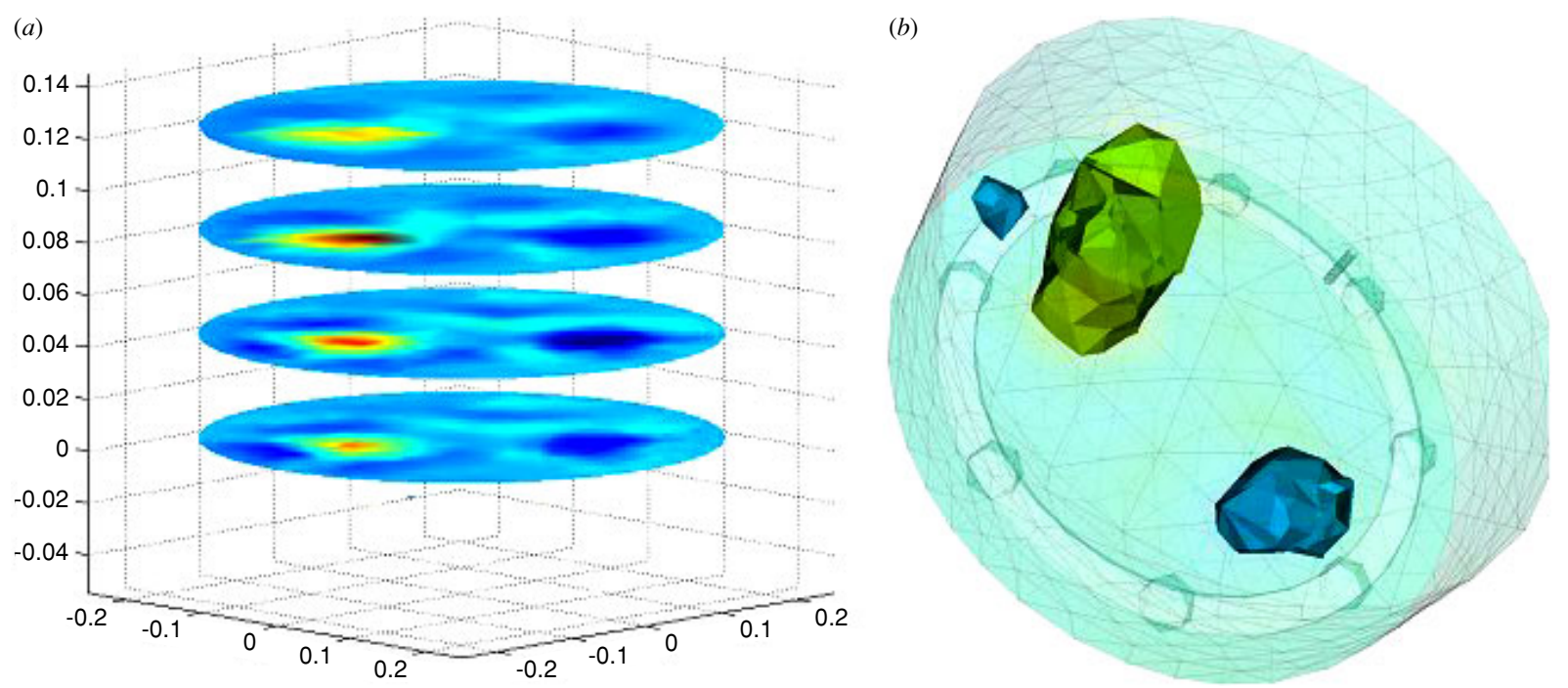

Figure 8. Reconstruction of two sponges in the aqueous system by the regularized method and using the stacked layered FEM model comprising approximately 4500 elements: (a) MatLab plot and (b) 3D image of the same data using MayaVi software. Isosurfaces generated automatically by MayaVi. The more conducting sponge is reconstructed on the LHS of $(a)$ and the same sponge is shown in the upper part of $(b)$. 


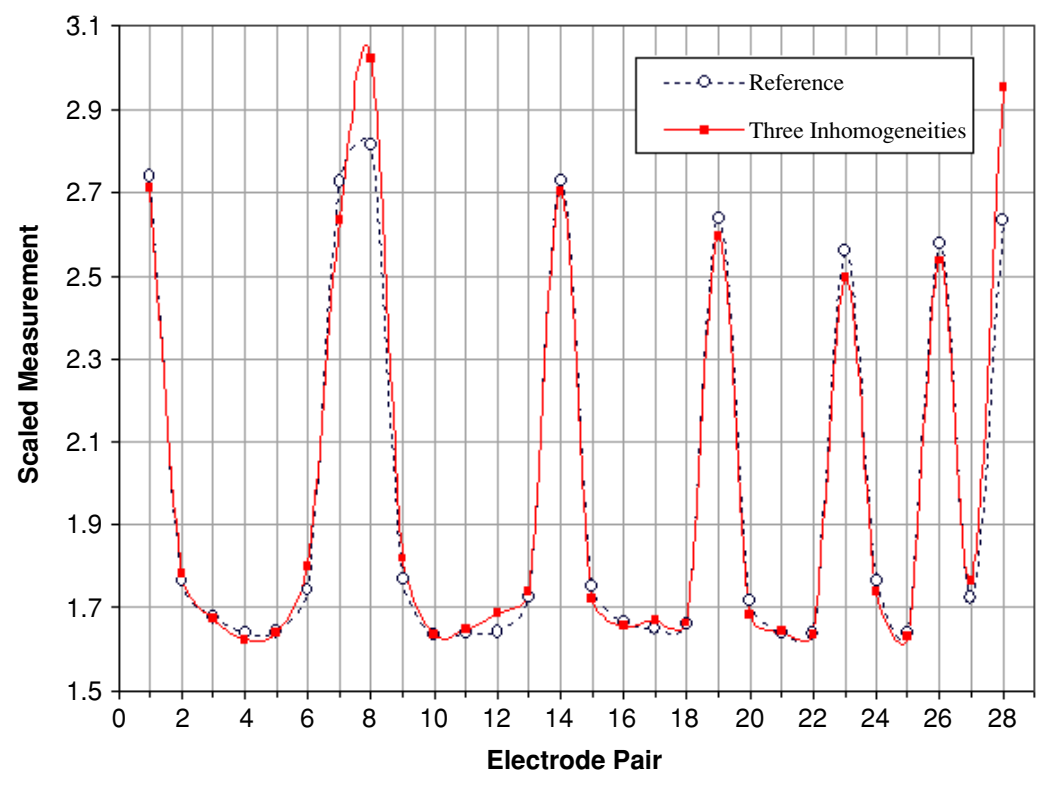

Figure 9. Measured voltage data scaled to unity injection current for the reference aqueous system and the addition of three inhomogeneities.

The figure shows both a MatLab plot and a 3D rendered image using MayaVi software [38]. The 3D plot uses a manually selected low level of opacity applied to a surface map, which describes the whole of the test filter to effectively view the complete internal volume and reveal two defined 3D isosurfaces representing the inhomogeneities. Importantly, it should be noted that the isosurfaces have been automatically determined by MayaVi as a result of the software searching for large discontinuities in the image data. The presence of such discontinuities shows the success of the regularized method in accurately reconstructing the actual sharp differences in conductivity between the phantoms and the background reference.

The regularized difference method has been successful in reconstructing three inhomogeneities in the aqueous system. Figure 9 shows the scaled voltage data for all 28 measurements for the reference case alongside the corresponding data after the introduction of the phantoms. Large measurement increases occur for electrode pairs 8 and 28, which correspond to current injections on electrodes 2 and 7 , whilst measuring on electrodes 3 and 8 respectively. This is to be expected, due to the close proximity of the two phantoms having significantly greater conductivities compared to the reference, which has the effect of increasing the potential field immediately surrounding the volume occupied by the inhomogeneities, and hence, the measurement increases. Conversely, the low conductivity phantom reduces the potential field in the immediate vicinity and this gives rise to reduced measurements for the injections referring to electrodes $3,4,5$ and 6 for measurements obtained on electrodes 4, 5, 6 and 7, as can be seen for pairs 14, 19, 23 and 26 in the figure. Although these changes are small, they are significant when compared with the measurement precision provided by the experimental system, which was confirmed by analysis of repeated measurements for the same phantom tests. Taking this into account, the data suggest that the lower conductivity phantom has given rise to more subtle changes but over a greater number of measurements compared with the higher conductivity phantoms.
The property of local inhomogeneities giving rise to global measurement changes, as a consequence of softfield perturbations, is the key to reconstructing the presence of multiple changes in conductivity. This is particularly significant for systems having only a small number of measurements, as is the case here, and the success of the regularized method in accurately reproducing the inhomogeneities from only 28 measurements can be clearly seen in figure 10. As for the double sponge phantom case, the reconstruction yields the appropriate locations and sizes for the effective conducting and less-conducting phantoms. Additionally, in common with the double phantom case, figure 10 shows small artificially reconstructed volumes of lower conductivity, which are not present in the physical systems. Such 'artefacts' are likely to occur for two reasons. Firstly, as a consequence of the soft-field in EIT, the actual conductivity distribution with respect to the measurements is essentially nonlinear, which has been approximated to a linear problem. Additionally, Tikhonov regularization is known to yield artefacts in the reconstruction [14]. Secondly, the absolute accuracy of the measurement system will determine the quality of the reconstructed image space. However, taking into account the small number of measurements, the level of artefacts can be considered minimal.

\section{Image reconstruction in the filter cake system}

The main purpose of developing EIT to monitor pressure filtration is to enable the identification of malformations and differences in localized drying of the filter cake. Figure 11 demonstrates the use of the regularized technique for the described filter cake experiment using an estimated background conductivity of $3.5 \mathrm{mS} \mathrm{m}^{-1}$ for image reconstruction. The removed cake material can be seen intensely, having been reconstructed as a more insulating region compared with the background filter-aid. This is a highly encouraging result, since compared with the 
(a)

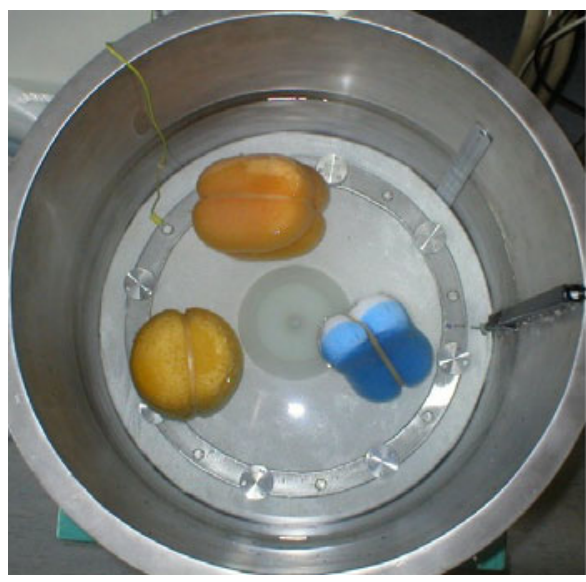

(b)

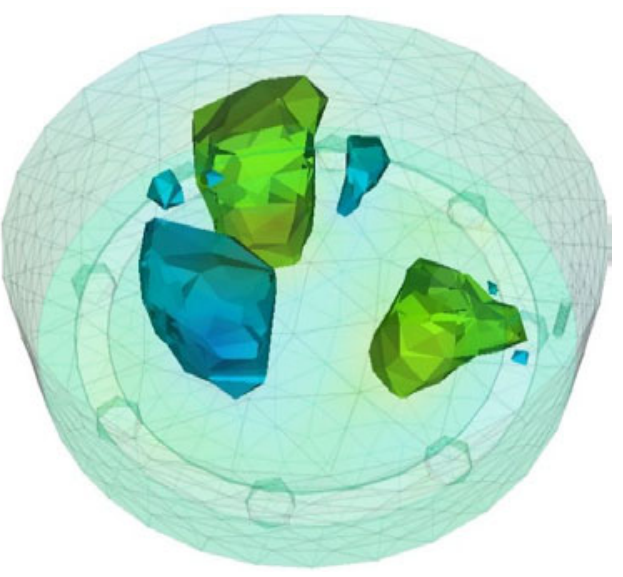

Figure 10. Reconstruction of three sponges in the aqueous system by the regularized method and using the stacked layered FEM model comprising approximately 4500 elements: $(a)$ experiment and (b) 3D image rendered using MayaVi software with automatic isosurfaces describing the sponge phantom locations. The lower conductivity sponge is shown in approximately the nine o'clock position and refers to phantom position 2 of figure $5(b)$.

(a)

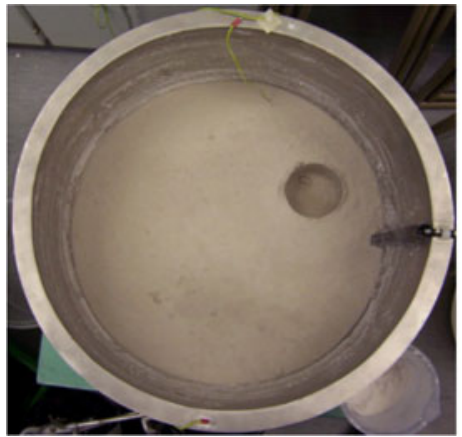

(b)

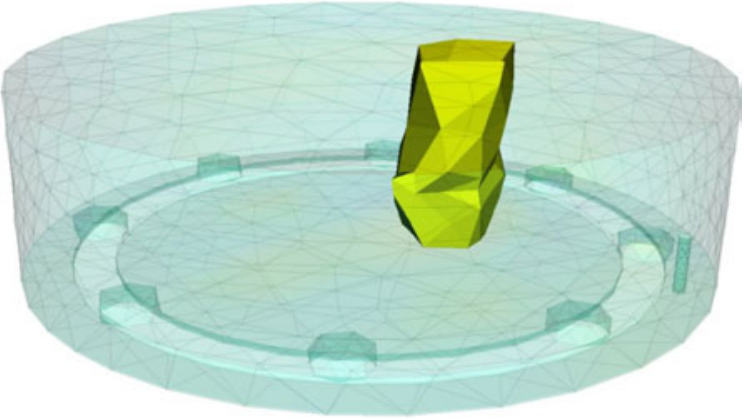

Figure 11. Reconstruction of the inhomogeneity in the filter aid system by the regularized method: $(a)$ experiment, $(b) 3 \mathrm{D}$ image rendered and showing the automatic isosurface found by MayaVi software.

aqueous system, the filter-aid background reference can be expected to be far less homogeneous due to differences in packing density and non-uniformity of drying during the cake formation process. This was confirmed by inspection of the raw measurement data and by taking several conductivity measurements in various positions from the upper region of the cake.

The predicted size and angular position of the exposed air volume are close to the actual physical inhomogeneity, however, the image reconstruction has underestimated the radial position of the cake-hole. This may be due to a number of factors. Firstly, due to memory allocation, the mesh describing the image reconstruction is comparatively coarse comprising less than 200 elements per stacked layer and this gives rise to a relatively small set of equations to be solved uniquely in three dimensions. The mathematics of the reconstruction process can only give a 'best-fit' to uniqueness, and for such a small system in $3 \mathrm{D}$, this may prove difficult to perform to a high level of accuracy. Consequently, this translates into a corresponding coarseness in 3D image quality, which is particularly true in this case, where no strong image filtering has taken place. Secondly, with only 28 measurements, the problem is highly under-determined, therefore, a high spatial resolution cannot be expected. Finally, the accuracy of the reconstruction depends upon the forward solution accuracy and, hence, the accuracy of the defined FEM model. In this case, the test vessel provides two directly grounded current sinks, firstly, to the hold-down bar and secondly, to the wall of the vessel itself. The electrode sensing plates are isolated from the central region of the holddown bar by PTFE components, which are relatively constant and physically well-defined and therefore, can be modelled accurately by FEM. However, due to machining tolerances of the bar and its positioning with respect to the vessel wall, the wall-to-electrode spacing can vary by approaching $\pm 5 \mathrm{~mm}$, which is almost $10 \%$ of the absolute electrode-towall spacing. Therefore, this spacing cannot be modelled to the same level of accuracy. However, a greater proportion of current will be lost to the bar since the absolute electrodeto-bar separation is significantly smaller compared with the electrode-to-wall separation. The two separations can be seen as providing two shared current paths to ground, which are analogous to a parallel impedance combination, where the impedances are determined by both the electrical properties of the filling medium and the electrode-to-bar and the electrodeto-wall spacings respectively. Therefore, the relative accuracy of the forward solution can be considered to depend not solely upon geometry effects but also on the filling medium itself. 
Consequently, it is difficult to quantify how these two issues combine in terms of overall image quality. This may begin to explain the better location accuracy for the higher conductivity aqueous experiments compared with the lower conductivity and less homogeneous powder-based system.

\section{Conclusions}

This work is the first to demonstrate true 3D images obtained using real measurements from an intrinsically safe EIT system applied to a metal-walled vessel. This has been achieved by performing calculations based on 3D forward solutions, which have resulted in providing good spatial accuracy and sensitivity to changes in conductivity. The results have demonstrated that the LBP method is highly sensitive to FEM modelling when applied to a metal-walled vessel and 3D image reconstruction. Additionally, it is concluded that a regularized difference method can accurately predict the location and size of multiple phantoms in true 3D from a single planar electrode arrangement yielding only a small number of measurements. This technique has the major advantage of providing good sensitivity without the need to create a highly defined FEM model of the vessel under study.

For the wetted powder-based system, the initial results strongly suggest that issues of non-uniformity and malformations within filter cakes can be imaged using the regularized difference method. Consequently, possibilities exist for providing qualitative $3 \mathrm{D}$ reconstructions capable of identifying differences occurring during individual process steps within a commercially operated pressure filter. Further advances in computing power and algorithm development should enable improved image reconstruction accuracy and sensitivity. Additionally, future work will concentrate on efforts to reduce the computational effort required to produce images by addressing meshing strategies. Furthermore, the image reconstruction methods will be applied to filter cake systems comprising wet and dry regions yielding subtle changes in conductivity as opposed to the present study, which has only considered more step-like differences.

\section{Acknowledgments}

This work has been supported under the ProceMon project as part of the DTI Link S3IA programme of the UK: project ref: YAF $8 / 2 / 3228$. The authors are most grateful to Dr Bill Lionheart and Dr Nick Polydorides of the UMIST Mathematics Department, for developing the indispensable 3D-EIDORS tool-suite, without which three-dimensional image reconstruction using 28 measurements would only remain possible on paper. Additionally, the authors would like to thank Mr Shahram Mohanna of the Industrial Process Tomography Group at UMIST for providing photographs of the experiments and instrumentation.

\section{References}

[1] Vlaev D, Wang M, Dyakowski T, Mann R and Grieve B D 2000 Detecting filter-cake pathologies in solid-liquid filtration: semi-tech scale demonstrations using electrical resistance tomography (ERT) Chem. Eng. J. 77 87-91
[2] Ruffino L, York T A, Mann R, Tiddy G, Tadthong O, Bonnet P and Grieve B D 2003 Potential of combined macro-scale tomography to monitor multiphase viscous fluid mixing $3 r d$ World Congress on Industrial Process Tomography (Banff, Canada)

[3] Hunt A, Pendleton J D and White R B 2003 A novel tomography flow analysis system 3rd World Congress on Industrial Process Tomography (Banff, Canada)

[4] Yuen E L, Mann R, York T A and Grieve B D 2001 Electrical resistance tomography imaging of a metal-walled solid-liquid filter 2nd World Congress on Industrial Process Tomography (Hannover, Germany)

[5] York T A, Smit Q, Davidson J L and Grieve B D 2003 An intrinsically safe electrical tomography system IEEE Int Symp. on Industrial Electronics (Rio de Janeiro, Brazil)

[6] York T A, Mazurkiewich L, Polydorides N, Mann R and Grieve B D 2003 Image reconstruction for pressure filtration using EIDORS 3D 3rd World Congress on Industrial Process Tomography (Banff, Canada)

[7] 1999 1st World Congress on Industrial Process Tomography (Buxton, UK) ISBN: 0853161933

[8] 2001 2nd World Congress on Industrial Process Tomography (Hannover, Germany) ISBN: 0853162247

[9] 2003 3rd World Congress on Industrial Process Tomography (Banff, Canada) ISBN: 0853162409

[10] Williams R A and Beck M S (ed) 1995 Process Tomography: Principles, Techniques and Applications 1st edn (Oxford: Butterworth-Heinemann)

[11] 1996 Special issue of Meas. Sci. Technol. on Electrical Tomography 7 (3)

[12] 2001 Special issue of Meas. Sci. Technol. on Electrical Tomography 12 (8)

[13] 2002 Special issue of Meas. Sci. Technol. on Electrical Tomography 13 (12)

[14] Player M A, van Weereld J, Hutchison J M S, Allen A R and Shang L 1999 An electrical impedance tomography algorithm with well-defined spectral properties Meas. Sci. Technol. 10 L9-L14

[15] Mann R, Dickin F J, Wang M, Dyakowski T, Forrest A E, Holden F J, Williams R A and Edwards R B 1997 Visualisation of stirred vessel mixing at plant scale using electrical resistance tomography 9th European Conf. on Mixing (Paris, France)

[16] Bolton G T, Hooper C W and Stitt E H 2003 Flow distribution and velocity measurement in a radial flow fixed bed reactor using electrical resistance tomography $3 r d$ World Congress on Industrial Process Tomography (Banff, Canada)

[17] Stanley S J, Wabo E, Mann R and Primrose K 2001 Dual-validation of miscible liquid mixing in a stirred vessel imaged by electrical resistance tomography (ERT) 2nd World Congress on Industrial Process Tomography (Hannover, Germany)

[18] Wang M 2001 Sensitivity theorem based on inverse solution using conjugate gradients methods for electrical impedance tomography 2nd World Congress on Industrial Process Tomography (Hannover, Germany)

[19] Gobel J C 1990 The three-dimensional inverse problem in electric current computed tomography PhD Thesis RPI

[20] Goble J C, Cheney M and Isaacson D 1992 Electrical impedance tomography in three dimensions Appl. Comput. Electromagn. Soc. J. 7 128-47

[21] Metherall P, Barber D C, Smallwood R H and Brown B H 1996 Three-dimensional electrical impedance tomography Nature 380 509-12

[22] Pinheiro P A T 1998 A three-dimensional image reconstruction algorithm for electrical resistance tomography PhD Thesis UMIST

[23] Vauhkonen M, Lionheart W R B, Heikkinen L M, Vauhkonen P J and Kaipio J P 2000 A Matlab package for the EIDORS project to reconstruct two-dimensional EIT images Physiol. Meas. 22 107-11 
[24] Polydorides N and Lionheart W R B 2002 A MatLab based toolkit for three-dimensional electrical impedance tomography: a contribution to the EIDORS project Meas. Sci. Technol. 13 1871-83

[25] Grieve B D 2002 On-line electrical impedance tomography for industrial batch processing $P h D$ Thesis UMIST

[26] Grieve B D, Smit Q, Mann R and York T A 2001 The application of electrical resistance tomography to a large volume production pressure filter 2nd World Congress on Industrial Process Tomography (Hannover, Germany)

[27] Grieve B D, Davidson J L, Mann R, Lionheart W R B and York T A 2003 Process compliant electrical impedance instrumentation for wide scale exploitation on industrial vessels 3rd World Congress on Industrial Process Tomography (Banff, Canada)

[28] Wang M, Dickin F J, Williams R A, Waterfall R C and Beck M C 1994 Electrical resistance tomography on metal walled vessel Int. Workshop on Process Tomography—A Strategy for Industrial Exploitation (Oporto, Portugal)

[29] Record P, Wang M and Dickin F 1995 Conducting boundary strategy: a new technique for medical EIT Physiol. Meas. 16 A249-55
[30] Wang M, Dickin F J and Williams R A 1994 Electrical resistance tomography of metal walled vessels and pipelines Electron. Lett. 30 771-3

[31] Cheng K S, Isaacson D, Newell J C and Gisser D G 1989 Electrode models for electric-current computed-tomography IEEE Trans. Biomed. Eng. 36 918-24

[32] Polydorides N 2002 Image reconstruction algorithms for soft-field tomography PhD Thesis UMIST

[33] Lionheart W R B 2001 Reconstruction algorithms for permittivity and conductivity imaging 2 nd World Congress on Industrial Process Tomography (Hannover, Germany)

[34] Lionheart W R B 2004 Developments in EIT reconstruction algorithms: pitfalls, challenges and recent developments Physiol. Meas. 25 125-42

[35] Yorkey T J, Webster J G and Tomkins W J 1987 Comparing reconstruction algorithms for electrical impedance tomography IEEE Trans. Biomed. Eng. 34 843-52

[36] Yorkey T J, Webster J G and Tomkins W J 1986 An optimal impedance tomographic reconstruction algorithm Ann. Int. Conf. IEEE Eng. Med. Biol. Soc. 8 339-42

[37] Netgen automatic mesh generator at http://www.hpfem.jku.at/ netgen/

[38] MayaVi scientific data visualizer at http://mayavi.sourceforge. net/ 